\title{
Aprendizagem Assistida por Pares: Uma Alternativa Pedagógica no Ensino de Competências a Estudantes de Medicina
}

\author{
Peer Assisted Learning: A Pedagogical Alternative of \\ Teaching Skills to Medical Students
}

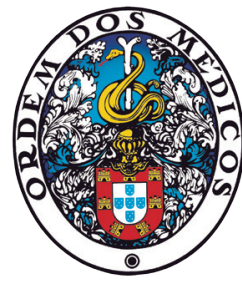

Joana Fernandes RIBEIRO $\bigotimes^{1}$, Manuel ROSETE², Andreia TEIXEIRA 3,4 , Hugo CONCEIÇÃO ${ }^{5}$, Lèlita SANTOS 6,7 Acta Med Port 2020 Nov;33(11):742-752 • https://doi.org/10.20344/amp.12125

RESUMO

Introdução: O treino de competências técnicas é fundamental para a prática clínica, mas pouco explorado no currículo médico pré-graduado. Neste contexto, o método de aprendizagem assistida por pares tem surgido como uma alternativa válida para contrariar esta insuficiência formativa. Neste estudo pretende-se avaliar o impacto, nos estudantes, de um programa de aprendizagem assistida por pares em técnicas cirúrgicas básicas, relativamente a competências técnicas e conhecimentos adquiridos.

Material e Métodos: Foram selecionados aleatoriamente 104 estudantes do terceiro ano de Medicina para participarem numa formação, lecionada por estudantes do quinto ano. Um total de 34 estudantes foram avaliados antes e após formação, através do instrumento Objective Structured Assessment of Technical Skills, composto por uma escala global de avaliação e uma lista de verificação específica de procedimentos. Estudantes do sexto ano (grupo de controlo) foram também submetidos a uma avaliação, sem formação. Os resultados do Objective Structured Assessment of Technical Skills obtidos pelos estudantes antes da formação versus após a formação foram comparados através dos testes de Wilcoxon e McNemar. Para comparar os resultados do Objective Structured Assessment of Technical Skills obtidos pelos estudantes após formação versus grupo de controlo foram utilizados os testes de Mann-Whitney, qui-quadrado e teste exato de Fisher.

Resultados: Na escala global de avaliação, os estudantes obtiveram uma pontuação após a formação $(29,5)$ significativamente superior à obtida antes da formação $(15,5 ; p$-value $<0,001)$, não tendo sido encontradas diferenças significativas entre após a formação e grupo de controlo ( $p$-value $=0,167)$. Na lista de verificação específica de procedimentos, os estudantes obtiveram uma evolução significativamente positiva em todos os parâmetros, sendo que estudantes após a formação obtiveram, maioritariamente, uma percentagem de realizações corretas bastante superior ao grupo de controlo.

Discussão: Os resultados obtidos demonstraram uma melhoria qualitativa e quantitativa significativa do conhecimento e competências técnicas dos estudantes, o que se encontra em consonância com a literatura encontrada.

Conclusão: Este programa de aprendizagem assistida por pares revelou-se promissor para a melhoria das competências técnicas cirúrgicas dos estudantes de medicina, com utilização de escassos recursos da faculdade e extensão a um número mais alargado de estudantes.

Palavras-chave: Cirurgia Geral/educação; Educação Médica Pré-graduada; Estudantes de Médicina; Grupo de Pares

\section{ABSTRACT}

Introduction: Technical skills training is fundamental for clinical practice although poorly emphasised in undergraduate medical curricula. In these circumstances, Peer Assisted Learning methodology has emerged as a valid alternative to overcome this insufficiency. The purpose of this study is to evaluate the impact on students of a Peer Assisted Learning program in basic surgical skills, regarding technical competences and knowledge improvement.

Material and Methods: A total of 104 randomly selected third year medical students participated in a workshop delivered by fifth year students. From that total, 34 students were assessed before and after the workshop, using the Objective Structured Assessment of Technical Skills instrument, that consists of a global rating scale and a procedure-specific checklist. Sixth year students (control group) were also assessed in their performance without participating in the workshop. Before workshop versus after workshop Objective Structured Assessment of Technical Skills results were compared using Wilcoxon and McNemar tests. After workshop versus control group Objective Structured Assessment of Technical Skills results were compared using Mann-Whitney, qui-squared test and Fisher's exact test.

Results: For the global rating scale, students obtained an after the workshop score (29.5) that was significantly higher than the before the workshop score $(15.5 ; p$-value $<0.001)$, but no significant differences were found between after the workshop and control group scores ( $p$-value $=0.167)$. For the procedure-specific checklist, $3^{\text {rd }}$ year students had a substantial positive evolution in all parameters and obtained higher rates of correct achievements compared to the control group.

Discussion: The final outcomes demonstrated a significant qualitative and quantitative improvement of knowledge and technical skills, which is in accordance with other literature.

Conclusion: This Peer Assisted Learning program revealed promising results concerning improvement of surgical skills in medical

1. Medicina Geral e Familiar. Unidade de Saúde Familiar Faria Guimarães. Agrupamentos de Centros de Saúde Porto Oriental. Porto. Portugal.

2. Serviço de Cirurgia Geral. Centro Hospitalar e Universitário de Coimbra. Coimbra. Portugal.

3. Departamento de Medicina da Comunidade, Informação e Decisão em Saúde. Faculdade de Medicina. Universidade do Porto. Porto. Portugal.

4. Centro de Investigação em Tecnologias e Serviços da Saúde. Universidade do Porto. Porto. Portugal.

5. Gabinete de Educação Médica. Faculdade de Medicina. Universidade de Coimbra. Coimbra. Portugal.

6. Serviço de Medicina Interna. Centro Hospitalar e Universitário de Coimbra. Coimbra. Portugal.

7. Clínica Universitária de Medicina Interna. Faculdade de Medicina. Universidade de Coimbra. Portugal.

$\triangle$ Autor correspondente: Joana Fernandes Ribeiro. jfrmed404@gmail.com

Recebido: 04 de abril de 2019 - Aceite: 10 de janeiro de 2020 | Copyright @ Ordem dos Médicos 2020 
students, with little staff faculty contribution and extension to a much broader number of students.

Keywords: Education, Medical, Undergraduate; General Surgery / education; Peer Group; Students, Medical

\section{INTRODUÇÃO}

As reformas estruturais que têm sido introduzidas na educação médica, fruto de uma maior preocupação com a abordagem holística do doente, têm resultado na diminuição da ênfase dada às competências técnicas (CT) no currículo médico pré-graduado. ${ }^{1-3}$ Alguns estudos sugerem que o ensino de CT, nas escolas médicas, é insuficiente, levando os estudantes a procurarem formação extracurricular ${ }^{1,3,4}$ e os recém-graduados a identificá-las como uma das áreas em que se consideram menos competentes (mediana de 2,8, numa escala de Likert 0 - 5). ${ }^{3}$ Contudo, o ensino de CT é fundamental para garantir um exercício autónomo e competente da profissão. ${ }^{1,2,5-8}$

Especificamente em cirurgia, algumas das competências nucleares, mencionadas nos documentos europeus são: técnica asséptica, manuseamento dos instrumentos cirúrgicos, técnicas de sutura e sua remoção. ${ }^{7-9}$ Relativamente às técnicas de sutura, que foram avaliadas num estudo de base nacional, ${ }^{3}$ os médicos portugueses recém-graduados têm uma autoperceção de baixa competência (mediana de 2,6, numa escala de Likert $0-5$ ). ${ }^{3}$

Transmitir conteúdos práticos requer esquemas educativos exigentes, com rácios estudante/tutor elevados ${ }^{10} \mathrm{e}$ disponibilidade alargada do corpo docente. ${ }^{11}$ No entanto, estes requisitos são limitações frequentemente encontradas pelas escolas médicas. O método de aprendizagem assistida por pares (AAP) surge como uma alternativa válida para contrariar esta insuficiência formativa. ${ }^{4,11,12}$

A AAP é um método de ensino, em que pessoas de grupos similares, não professores, se auxiliam mutuamente a aprender e aprendem a ensinar. ${ }^{13} \mathrm{~A}$ sua utilização no ensino médico iniciou-se na década de 70, nos Estados Unidos da América $^{14}$, sendo considerado um bom método de promoção do ensino em pequenos grupos, com uso sustentável de recursos e atingindo uma população mais alargada. ${ }^{13,15,16}$ Desde então tem vindo a ser incorporado no currículo médico, em inúmeras universidades a nível mundial, em diversas áreas e, com resultados positivos para formandos/formadores e para o currículo médico. ${ }^{12-20} \dot{E}$ um método comprovado no ensino da anatomia humana, ${ }^{12} \mathrm{com}$ resultados igualmente promissores no treino do exame físico. ${ }^{12}$ Recentemente, tem-se assistido a um incremento da sua aplicação ao ensino de CT, nomeadamente técnicas cirúrgicas básicas (TCB), em escolas médicas do Reino Unido como Keele, Exeter e Cardiff, e de São Paulo, no Brasil.4,11,21,22 Os resultados obtidos têm demonstrado meIhoria significativa das competências dos estudantes em TCB, de forma sustentável e pouco dispendiosa. ${ }^{4,11,21,22}$

Algumas das vantagens documentadas da AAP incluem: congruência cognitiva e social, ${ }^{23}$ ambiente propício à aprendizagem, reforço do conhecimento dos estudantes que ensinam, aumento da motivação, treino de liderança e confiança, maior envolvimento dos estudantes no proces- so de aprendizagem e de desenvolvimento e, preparação dos estudantes para o futuro papel enquanto educadores. ${ }^{12,13,15,16}$

O objetivo principal deste trabalho é avaliar o impacto de um programa de $\mathrm{AAP}$ em TCB, relativamente à melhoria do conhecimento e da CT em estudantes de Medicina. Este programa insere-se na modalidade de ensino por quase-pares (Near-Peer Teaching), que consiste no ensino de estudantes de níveis iniciais de um curso, por estudantes de níveis mais avançados do mesmo programa de estudos, com diferença de um ou mais anos. ${ }^{15}$ Para atingir o objetivo principal, consideram-se três objetivos específicos: avaliar a perceção dos estudantes formandos relativamente ao programa de AAP; comparar o nível de conhecimento e CT antes e após programa de AAP e, comparar o nível de conhecimento e CT após programa de AAP com o nível de conhecimento e CT de estudantes finalistas.

\section{MATERIAL E MÉTODOS}

O Gabinete de Educação Médica (GEM) da Faculdade de Medicina da Universidade de Coimbra (FMUC) e o Núcleo de Estudantes de Medicina da Associação Académica de Coimbra (NEM/AAC), no ano letivo 2015/2016, estruturaram um programa piloto de AAP, com base no guia $n^{\circ} 30$ da Association for Medical Education in Europe. ${ }^{15}$ Este programa consistiu numa sessão formativa sobre TCB a estudantes do terceiro ano, por estudantes do quinto ano do Mestrado Integrado em Medicina (MIM). Os conhecimentos sobre TCB dos estudantes do terceiro ano foram avaliados antes da sessão formativa ( $\mathrm{AnF}$ ) e, após a sessão formativa $(\mathrm{ApF})$, por um examinador. Além disto, avaliou-se os conhecimentos sobre TCB de um grupo de estudantes do sexto ano do MIM [grupo de controlo (GC)], com o objetivo de comparar com os conhecimentos avaliados ApF dos estudantes do terceiro ano que frequentaram a sessão.

\section{Participantes}

O programa de APP envolveu três tipos de estudantes participantes: formadores, formandos e controlo.

Os formadores foram 12 estudantes inscritos no quinto ano do MIM da FMUC, no ano letivo 2015/2016. Optou-se pelo quinto ano por ser um ano avançado da formação médica, mas com maior disponibilidade de tempo, por comparação com os estudantes do sexto ano. Estes foram recrutados através da criação de uma bolsa de voluntários, conforme sugerido por Wadoodi e Crosby. ${ }^{24}$ Receberam uma formação de 16 horas, dividida proporcionalmente em duas componentes: uma pedagógica, da responsabilidade do GEM e uma técnica, lecionada por dois médicos internos experientes (dos terceiros e quarto anos do internato de seis anos) da especialidade de Cirurgia Geral do Centro Hospitalar e Universitário de Coimbra, EPE. O conteúdo 
desta componente técnica baseou-se nas temáticas gerais do curso do Royal College of Surgeons of England - Surgical Skills for Students. ${ }^{25}$ Os formadores foram informados dos objetivos da sessão e dos conteúdos a abordar, mas a implementação da sessão e as metodologias utilizadas foram da sua total responsabilidade.

Os formandos foram estudantes inscritos no terceiro ano do MIM, do mesmo ano letivo, por ser o último ano pré-clínico e onde já está planificado o ensino de procedimentos práticos, nomeadamente sutura. O NEM/AAC possuía uma listagem com esses 258 estudantes. A estes estudantes foram aplicados dois critérios de exclusão: terem participado em formações extracurriculares semelhentes e/ou terem um outro curso superior numa área afim. Os critérios de exclusão reduziram a lista para 224 formandos, que foram ordenados por ordem alfabética de nome e, a cada um deles, atribuído um número inteiro entre 1 e 224. Posteriormente, foram selecionados aleatoriamente 130 estudantes, aproximadamente $58 \%$ da população em estudo (o cálculo do tamanho da amostra é descrito na secção Tamanho da Amostra), através de um gerador de números aleatórios, disponível em linha. ${ }^{26} \mathrm{~A}$ estes estudantes selecionados, foi direcionado, através dos delegados de turma e, por escrito, um convite de participação e uma breve descrição do programa de AAP, sendo que 104 estudantes aceitaram participar.

Os estudantes que aceitaram participar receberam a sessão formativa de TCB. No entanto, por limitações logísticas, condicionadas pelo fator tempo e pela disponibilidade do examinador, não seria possível avaliar antes e após a sessão um número tão elevado de estudantes, tendo-se estipulado que a avaliação de conhecimentos só seria aplicada a um subconjunto destes estudantes. Assim, voltou-se a ordenar, por ordem alfabética de nome e, a atribuir a cada um deles, sequencialmente, um número inteiro entre 1 e 104. Posteriormente, foram selecionados aleatoriamente 40 estudantes [grupo de avaliação (GA)], utilizando uma vez mais o gerador de números aleatórios. A estes estudantes, antes e depois da sessão formativa, foi aplicado um instrumento de medida, Objective Structured Assessment of Technical Skills (OSATS), ${ }^{27-30}$ de modo a aferir os conhecimentos sobre TCB.

O GC foi constituído por estudantes do sexto ano com o estágio de cirurgia concluído, tendo-lhe sido aplicados os mesmos critérios de exclusão aplicados aos alunos de terceiro ano. Para a seleção destes estudantes, foi também criada uma bolsa de voluntários, tendo-se obtido um grupo de 20 estudantes. O GC não recebeu sessão formativa, apenas foram avaliados relativamente aos conhecimentos de TCB, através do OSATS.

A todos os participantes foi garantido o anonimato e todos deram o consentimento informado verbalmente. O estudo foi submetido e aprovado pela Faculdade de Medicina da Universidade de Coimbra, de acordo com as regras estabelecidas para os trabalhos finais de Mestrado Integrado em Medicina, pelo que não foi submetido a Comissão de Ética.

\section{Sessão formativa}

No total, foram realizadas cinco sessões formativas. Cada uma abrangeu oito estudantes, durou três horas e foi ministrada por dois estudantes formadores, num rácio formador:formando de 1:4, tal como Dubrowski e MacRae sugerem. ${ }^{31}$ Cada sessão foi composta por uma exposição teórica e por uma componente prática em modelo de sutura suíno. Os conteúdos abordados na sessão formativa foram baseados nos objetivos preconizados para a formação pré-graduada: técnica assética (lavagem cirúrgica das mãos, utilização de proteção individual, colocação de luvas esterilizadas, desinfeção de ferida cirúrgica e não cirúrgica), princípios de encerramento de feridas, fios de sutura, instrumentos cirúrgicos e seu manuseamento, técnica de sutura simples, tempo de remoção de sutura e técnica de remoção de pontos e agrafos.

\section{Avaliação objetiva do desempenho}

Cada elemento do GA foi sujeito a uma prova prática de TCB, também em modelo de sutura suíno, com a duração de 10 minutos e, assistida por um examinador (médico interno do quinto ano do internato de seis anos de Cirurgia Geral). O examinador avaliou os desempenhos, tendo por base $o$ instrumento OSATS, ${ }^{27-30}$ validado para internos de cirurgia ${ }^{27}$ e traduzido para a língua portuguesa e aplicado a estudantes de medicina por Denadai et al. ${ }^{29}$ É constituído por duas componentes: uma escala global de avaliação para procedimentos cirúrgicos (EGA) e uma lista de verificação específica de procedimentos (LVEP).

A EGA permite avaliar aspetos qualitativos globais do desempenho de TCB, sendo constituída por oito itens, medidos por escalas de Likert 1 - 5: respeito pelo tecido (RT), tempo no movimento (TM), manuseamento dos instrumentos (MI), treino de sutura (TS), fluência do procedimento (FP), conhecimento do procedimento, (CP), produto final (PF) e performance geral (PG). A pontuação total da escala, PT, varia de 8 a 40 pontos, mas, por definição, o avaliando é considerado competente na técnica se obtiver uma pontuação não inferior a 24 pontos. ${ }^{28}$

A LVEP é menos dependente do examinador e indica se realizado corretamente/não realizado corretamente para cada um dos seguintes parâmetros: técnica assética (TA), limpeza centrífuga da ferida duas vezes (LCF2x), escolha apropriada do tipo de fio de sutura (EATFS), colocação de agulha no porta-agulhas (CAPA), entrada e saída perpendicular do tecido (ESPT), perfuração do tecido não inferior a $0,5 \mathrm{~cm}$ (PTNI), técnica de nó com porta-agulhas (TNPA), técnica de 'nó de cirurgião' (TNC), corte do fio de sutura com margens adequadas (CFSMA) e técnica de remoção da sutura (TRS).

Para a avaliação AnF, os formandos não tiveram qualquer tipo de preparação extracurricular, pelo que se apresentaram com os conhecimentos aquiridos ao longo dos primeiros três anos de curso. Além disso, desconheciam o conteúdo da prova prática e a estrutura da avaliação. Considerou-se que estavam todos em condições equivalentes, uma vez que eram todos estudantes do mesmo 
ano letivo e curricular e da mesma instituição de ensino. A avaliação ApF foi idêntica na estrutura, mas variou o conteúdo, de modo a tirar partido do fator surpresa. Entre a primeira avaliação e a sessão formativa decorreram três semanas, por motivos relacionados com a disponibilidade dos envolvidos. A segunda avaliação ocorreu um mês após a sessão formativa, de modo a que não fosse apenas avaliado o impacto imediato, mas também a retenção do conhecimento (ou da CT). ${ }^{32}$

Não foi dado feedback verbal durante as avaliações. O examinador não teve acesso a informação que identificasse os estudantes e foram utilizadas folhas de avaliação diferentes nos dois momentos.

\section{Questionário de autoperceção}

No final da sessão, os formandos foram convidados a preencher um questionário de satisfação (ver Apêndice 1: https://www.actamedicaportuguesa.com/revista/index.php/ amp/article/view/12125/Apendice_01.pdf). Este questionário teve como objetivo avaliar a perceção dos estudantes sobre a sessão formativa, nomeadamente o impacto que esta teve na confiança, motivação, conhecimento e CT dos formandos e, ainda, avaliar alguns parâmetros qualitativos da sessão, nomeadamente: competências/conhecimentos dos formadores, tamanho dos grupos de trabalho, ambiente de aprendizagem, método de ensino e recursos didáticos utilizados. As questões foram maioritariamente fechadas, com opções de resposta em escalas de Likert 1 - 5 (1 "discordo totalmente"; 5 - "concordo totalmente") ou dicotómicas $\operatorname{sim} /$ não.

Uma vez que os os autores não encontraram, na literatura, nenhum questionário validado que fosse de encontro aos objetivos pretendidos, procederam à elaboração deste questionário e efetuaram um pré-teste a uma amostra de conveniência de 10 pessoas, não tendo sido diagnosticado nenhum erro nem na formatação nem na interpretação.

\section{Tamanho da amostra}

Dos 224 constituintes da população em estudo, foram selecionados 130 participantes. Este tamanho de amostra foi determinado para proporções, considerando: nível de confiança $=95 \%$, proporção amostral $=50 \%$ (cenário conservativo) e erro amostral $=5,6 \%$. No entanto, dos 130 selecionados, apenas 104 aceitaram participar no programa, correspondendo assim a um erro amostral de, aproximadamente, $7,1 \%$. Para o estudo do efeito da sessão formativa foram selecionados aleatoriamente 40 estudantes destes 104, sem cálculo prévio do tamanho amostral. A justificação deste tamanho deveu-se apenas a limitações logísticas da operacionalização do estudo. De forma a contornar esta limitação, nas análises efetuadas aos efeitos da sessão, são apresentadas as respetivas magnitudes de efeito.

\section{Métodos estatísticos}

A análise de dados foi efetuada com recurso ao Microsoft Excel 2016 e ao SPSS v.25.

Variáveis qualitativas nominais são descritas pelas res- petivas frequências absolutas (n) e relativas (\%). Variáveis qualitativas ordinais e quantitativas não normais são descritas pela mediana (Med) e pelo respetivo intervalo inter-quartil $\left[Q_{1} ; Q_{3}\right]$, em que $Q_{1}$ representa o primeiro quartil e $Q_{3}$ representa o terceiro quartil. A normalidade das variáveis quantitativas foi verificada por observação dos respetivos histogramas.

Para comparar duas distribuições emparelhadas utilizou-se o teste de Wilcoxon, no caso de variáveis ordinais ou quantitativas não normais e o teste de McNemar, no caso de variáveis dicotómicas.

Para comparar duas distribuições independentes de variáveis quantitativas não normais utilizou-se o teste de Mann-Whitney.

Para verificar a independência de variáveis dicotómicas utilizou-se o teste do qui-quadrado ou o teste exato de Fisher, quando o teste qui-quadrado não cumpria os requisitos.

As respetivas magnitudes de efeito $(m e)$ foram determinadas de acordo com o teste em causa: Wilcoxon - razão entre a estatística de teste padronizada e a raíz quadrada do número de observações; McNemar $-p-0,5$, sendo $p$ o máximo entre $b /(b+c)$ e $c /(b+c)$, em que b e c correspondem às células discordantes numa tabela de dupla entrada; Mann-Whitney - razão entre a estatística de teste padronizada e a raíz quadrada do número total de indivíduos; Qui-quadrado/Exato de Ficher - coeficiente Phi. Magnitudes de efeito de 0,1 são pequenas, de 0,3 são médias e acima de 0,5 são grandes.

Valores de $p \leq 0,05$ foram considerados significativos.

\section{RESULTADOS}

A sessão formativa de TCB foi frequentada pelos 104 estudantes do terceiro ano do MIM que aceitaram o convite de participar no programa de AAP.

A Fig. 1 descreve o desenho de estudo deste trabalho de investigação.

\section{Questionário de autoperceção}

Dos 104 estudantes que aceitaram participar no programa de AAP, 89 responderam ao questionário de avaliação da sessão (ver Apêndice 1: https://www.actamedicaportuguesa.com/revista/index.php/amp/article/view/12125/ Apendice_01.pdf). Relativamente à idade, 54 (60,7\%) tinham idade entre 19 e 21 anos, 32 (36\%) tinham entre 22 e 24 anos e $3(3,4 \%)$ tinham 25 ou mais anos. No que concerne a contactos anteriores com a técnica de sutura, $51(57,3 \%)$ responderam que sim, que já tinham tido contacto em alguma unidade curricular. Destes 51, 44 (86,3\%) responderam que o número de horas aproximado de contacto com a técnica foi de 1 a 3 horas, seis $(11,8 \%)$ responderam de 4 a 9 horas e apenas um (2\%) respondeu mais de 9 horas. Relativamente a contacto anterior com noções de assépsia e limpeza de feridas, 59 (66,3\%) responderam negativamente. Dos que responderam afirmativamente, 27 (90\%) responderam que o número de horas aproximado de aprendizagem na técnica foi de 1 a 3 horas e os restantes 


\begin{tabular}{|c|c|c|}
\hline $\begin{array}{l}\text { Alunos do } 3^{\circ} \text { ano } \\
(n=104)\end{array}$ & & $\begin{array}{l}\text { Alunos do } 6^{\circ} \text { ano } \\
\text { (grupo de controlo) }\end{array}$ \\
\hline $\begin{array}{l}\text { Avaliação pré-sessão formativa } \\
\qquad(n=40)\end{array}$ & \multirow[b]{2}{*}{$\mathrm{n}=55$} & $n=20$ \\
\hline Escala global de avaliação ${ }^{1}$ & & \\
\hline \multicolumn{2}{|c|}{$\begin{array}{l}\text { Três horas de sessão formativa em TCB } \\
\text { Racio formador:formando } 1: 4 \\
\text { Componente prática + teórica } \\
\text { Modelo suíno }\end{array}$} & \\
\hline $\begin{array}{l}\text { Avaliação pós-sessão formativa } \\
\qquad(n=34)\end{array}$ & $\begin{array}{c}n=89 \\
(34+55)\end{array}$ & Avaliação única \\
\hline $\begin{array}{l}\text { Escala global de avaliação }{ }^{1} \\
\text { Lista de verificação }{ }^{1} \\
\text { Questionário de autoperceção }\end{array}$ & $\begin{array}{l}\text { Questionário de } \\
\text { autoperceção }\end{array}$ & $\begin{array}{l}\text { Escala global de avaliaçãó } \\
\text { Lista de verificação }^{1}\end{array}$ \\
\hline
\end{tabular}

Figura 1 - Desenho do estudo

1: Componentes do instrumento Objective Structured Assessment of Technical Skills (OSATS) validado para técnicas cirúrgicas AAP: Aprendizagem assistida por pares; TCB: Técnicas cirúrgicas básicas; MIM: Mestrado integrado em Medicina

três (10\%) responderam de 4 a 9 horas. Dos 89,64 (71,9\%) discordaram que receberam formação suficiente em técnica de sutura e $61(68,5 \%)$ discordaram que receberam formação suficiente em noções de assépsia e limpeza de feridas.

As respostas às perguntas sobre o impacto e a qualidade da sessão podem ser consultadas na Tabela 1. Relativamente ao impacto, a maioria dos estudantes considerou-o positivo: na CT (84; $94,4 \%)$; no conhecimento (82; 92,1\%), na confiança $(82 ; 92,1 \%)$ e na motivação $(81 ; 91 \%)$ no desempenho das técnicas abordadas (Tabela 1). Relativamente à qualidade, a maioria avaliou como alta/muito alta a qualidade associada a cada um dos parâmetros.

Todos os estudantes referiram que voltariam a participar numa sessão lecionada por estudantes de anos mais avançados e $84(94,4 \%)$ responderam que considerariam a possibilidade de, no futuro, participarem como formadores num programa de AAP.

Tabela 1 - Respostas dos 89 estudantes do terceiro ano do MIM que preencheram o questionário de avaliação à sessão formativa

\begin{tabular}{|c|c|c|c|c|c|c|}
\hline & 1 & 2 & 3 & 4 & 5 & $\operatorname{Med}\left[Q_{1} ; Q_{3}\right]$ \\
\hline \multicolumn{7}{|l|}{ Impacto da sessão formativa } \\
\hline $\begin{array}{l}\text { Sinto que o workshop foi útil para desenvolver a } \\
\text { minha competência técnica nas áreas abordadas. }\end{array}$ & $0(0 \%)$ & $0(0 \%)$ & $5(5,6 \%)$ & $37(41,6 \%)$ & $47(52,8 \%)$ & $5[4 ; 5]$ \\
\hline $\begin{array}{l}\text { Sinto que o workshop foi útil para desenvolver o meu } \\
\text { conhecimento teórico nas áreas abordadas. }\end{array}$ & $0(0 \%)$ & $0(0 \%)$ & $7(7,9 \%)$ & $43(48,3 \%)$ & $39(43,8 \%)$ & $4[4 ; 5]$ \\
\hline $\begin{array}{l}\text { Após o workshop senti-me mais confiante para } \\
\text { executar as tarefas que me foram solicitadas. }\end{array}$ & $0(0 \%)$ & $1(1,1 \%)$ & $6(6,7 \%)$ & $52(58,4 \%)$ & $30(33,7 \%)$ & $4[4 ; 5]$ \\
\hline $\begin{array}{l}\text { Sinto que, após o workshop, fiquei mais motivado } \\
\text { para aprender mais sobre técnicas cirúrgicas/sutura. }\end{array}$ & $0(0 \%)$ & $0(0 \%)$ & $8(9 \%)$ & $42(47,2 \%)$ & $39(43,8 \%)$ & $4[4 ; 5]$ \\
\hline $\begin{array}{l}\text { Sinto que fui ensinado num ambiente descontraído } \\
\text { que potenciou a minha aprendizagem. }\end{array}$ & $0(0 \%)$ & $0(0 \%)$ & $4(4,5 \%)$ & $26(29,2 \%)$ & $59(66,3 \%)$ & $5[4 ; 5]$ \\
\hline \multicolumn{7}{|l|}{ Qualidade da sessão formativa } \\
\hline Competência/ conhecimento dos formadores. & $0(0 \%)$ & $0(0 \%)$ & $3(3,4 \%)$ & $47(52,8 \%)$ & $39(43,8 \%)$ & $4[4 ; 5]$ \\
\hline Tamanho dos grupos de trabalho. & $0(0 \%)$ & $2(2,2 \%)$ & $4(4,5 \%)$ & $33(37,1 \%)$ & $50(56,2 \%)$ & $5[4 ; 5]$ \\
\hline Ambiente de aprendizagem. & $0(0 \%)$ & $0(0 \%)$ & $0(0 \%)$ & $30(33,7 \%)$ & $59(66,3 \%)$ & $5[4 ; 5]$ \\
\hline Método de ensino. & $0(0 \%)$ & $0(0 \%)$ & $4(4,5 \%)$ & $36(40,4 \%)$ & $49(55,1 \%)$ & $5[4 ; 5]$ \\
\hline Recursos didáticos. & $1(1,1 \%)$ & $0(0 \%)$ & $11(12,4 \%)$ & $39(43,8 \%)$ & $38(42,7 \%)$ & $4[4 ; 5]$ \\
\hline
\end{tabular}

Na secção sobre o impacto da sessão formativa, 1: Discordo totalmente; 5: Concordo totalmente. Na secção sobre a qualidade da sessão formativa, 1: Muito baixa; 2: Baixa; 3: Média; 4: Alta; 5: Muito alta. 


\section{Avaliação objetiva do desempenho}

O GA era constituído por 40 estudantes e, no primeiro momento de avaliação compareceram todos. No entanto, ao segundo momento de avaliação, apenas compareceram 34 , uma vez que seis estudantes passaram a preencher critérios de exclusão. Assim, os resultados apresentados, na Tabela 2, são referentes aos 34 estudantes que completaram os dois momentos de avaliação: AnF e ApF. Destes $34,23(67,6 \%)$ eram do sexo feminino.

Relativamente à avaliação obtida pela EGA, os estudantes obtiveram uma PT mediana de 15,5 pontos AnF e uma PT mediana de 29,5 pontos ApF (Tabela 2), verificando-se um aumento estatisticamente significativo ( $p$-value $<0,001)$ de 14 pontos, refletindo-se numa grande me $(0,62)$. Aliás, verificou-se um aumento estatisticamente significativo dos valores medianos, em todos os itens da EGA, sendo que todos apresentam me grandes. Na avaliação efetuada AnF, apenas um estudante obteve uma pontuação de 24 pontos, ou seja, apenas um $(2,9 \%)$ estudante era considerado competente ${ }^{29}$ na técnica de sutura simples. $\mathrm{Na}$ avaliação efetuada ApF, $30(88,2 \%)$ estudantes obtiveram uma pontuação superior a 24 pontos, logo verificou-se um aumento no número de estudantes considerados competentes.

Relativamente à LVEP, obteve-se uma evolução positiva, estatisticamente significativa, no desempenho dos estudantes para todos os parâmetros (Tabela 2). ApF, os parâ- metros LCF2x e TNPA foram realizados corretamente pelos 34 estudantes, enquanto que AnF, estas técnicas tinham sido realizadas corretamente por nove $(26,5 \%)$ e $17(50 \%)$, respetivamente. Apesar de todos os parâmetros apresentarem uma percentagem de realizações corretas, significativamente superior $A p F$, os parâmetros que registaram uma maior evolução foram: TA - diferença $=28(82,4 \%) ; m e=$ 0,$5 ; p$-value < 0,001; LCF2x - diferença $=25(73,5 \%) ; m e$ $=0,5 ; p$-value <0,001; ESPT - diferença $=24(70,6 \%) ; m e$ $=0,5 ; p$-value $<0,001 ;$ TNC - diferença $=24(70,6 \%) ; m e$ $=0,46 ; p$-value <0,001 e TNPA - diferença $=17(50 \%)$; $m e=0,5 ; p$-value $<0,001$. Os parâmetros que registaram uma menor evolução foram: CAPA - diferença $=16$ $(47,1 \%) ; m e=0,36 ; p$-value $=0,001 ;$ EATFS - diferença $=$ $14(41,2 \%) ; m e=0,35 ; p$-value $=0,003 ;$ CFSMA - diferença $=13(38,2 \%) ; m e=0,34 ; p$-value $=0,004 ;$ PTNI - diferença $=11(32,4 \%) ; m e=0,24 ; p$-value $=0,035$ e TRS - diferença $=10(29,4 \%) ; m e=0,31 ; p$-value $=0,021$. O parâmetro com menor número de realizações corretas $\mathrm{ApF}$ foi o parâmetro TRS, com $18(52,9 \%)$ estudantes a realizá-lo corretamente.

\section{Comparação de desempenho com o GC}

O GC consistiu em 20 estudantes do sexto ano. Os resultados obtidos por este grupo foram comparados com os resultados obtidos pelo GAApF (Tabela 3).

Relativamente à avaliação através da EGA, apenas o

Tabela 2 - Avaliação dos 34 estudantes do terceiro ano do MIM que se submeteram aos dois momentos de avaliação: antes e após a sessão formativa

\begin{tabular}{|c|c|c|c|c|}
\hline & $\begin{array}{c}\text { Antes da sessão } \\
n=34\end{array}$ & $\begin{array}{c}\text { Após a sessão } \\
n=34\end{array}$ & $p$-value & $\begin{array}{c}\text { Magnitude } \\
\text { de efeito }\end{array}$ \\
\hline \multicolumn{5}{|l|}{ EGA, Med $\left[Q_{1} ; Q_{3}\right]$} \\
\hline Respeito pelo tecido & $2[1,75 ; 2]$ & $4[3 ; 4]$ & $<0,001^{a, *}$ & 0,60 \\
\hline Tempo no movimento & $2[1 ; 2]$ & $3[3 ; 4]$ & $<0,001^{a, *}$ & 0,59 \\
\hline Manuseamento dos instrumentos & $2[1 ; 2]$ & $3[3 ; 4]$ & $<0,001^{a, *}$ & 0,59 \\
\hline Treino de sutura & $2[1 ; 2]$ & $4[3 ; 4]$ & $<0,001^{a, *}$ & 0,60 \\
\hline Fluência do procedimento & $2[1 ; 2,25]$ & $4[3 ; 4]$ & $<0,001^{a, *}$ & 0,60 \\
\hline Conhecimento do procedimento & $2[1 ; 3]$ & $4[3 ; 4]$ & $<0,001^{a, *}$ & 0,59 \\
\hline Produto final & $2[1 ; 3]$ & $4[3 ; 4]$ & $<0,001^{a, *}$ & 0,60 \\
\hline Performance geral & $2[1 ; 3]$ & $4[3 ; 4]$ & $<0,001^{a, *}$ & 0,60 \\
\hline Pontuação total & $15,5[9 ; 19]$ & $29,5[26 ; 32]$ & $<0,001^{a, *}$ & 0,62 \\
\hline \multicolumn{5}{|l|}{ Lista de verificação específica de procedimento, $\mathrm{n}(\%)$} \\
\hline Técnica asséptica & $1(2,9 \%)$ & $29(85,3 \%)$ & $<0,001^{\mathrm{b}, *}$ & 0,5 \\
\hline Limpeza centrífuga da ferida duas vezes & $9(26,5 \%)$ & $34(100 \%)$ & $<0,001^{\mathrm{b}, *}$ & 0,5 \\
\hline Escolha apropriada do tipo de fio de sutura & $7(20,6 \%)$ & $21(61,8 \%)$ & $0,003^{b, *}$ & 0,35 \\
\hline Colocação de agulha no porta-agulhas & $13(38,2)$ & $29(85,3)$ & $0,001^{\mathrm{b}, *}$ & 0,36 \\
\hline Entrada e saída perpendicular do tecido & $9(26,5 \%)$ & $33(97,1 \%)$ & $<0,001^{\mathrm{b}, *}$ & 0,50 \\
\hline Perfuração do tecido não inferior a $0,5 \mathrm{~cm}$ & $16(47,1 \%)$ & $27(79,4 \%)$ & $0,035^{b, *}$ & 0,24 \\
\hline Técnica de nó com porta-agulhas & $17(50 \%)$ & $34(100 \%)$ & $<0,001^{\mathrm{b}, *}$ & 0,50 \\
\hline Técnica de "nó de cirurgião" & $8(23,5 \%)$ & $32(94,1 \%)$ & $<0,001^{\mathrm{b}, *}$ & 0,46 \\
\hline Corte do fio de sutura com margens adequadas & $9(26,5 \%)$ & $22(64,7 \%)$ & $0,004^{b, *}$ & 0,34 \\
\hline Técnica de remoção da sutura & $8(23,5 \%)$ & $18(52,9 \%)$ & $0,021^{b, *}$ & 0,31 \\
\hline
\end{tabular}

a: teste de Wilcoxon; b: teste de McNemar.

*: significativo a $5 \%$ 
Tabela 3 - Comparação entre as avaliações do grupo de avaliação (após a sessão formativa) e o grupo de controlo

\begin{tabular}{|c|c|c|c|c|}
\hline & $\begin{array}{c}\text { Grupo de avaliação } \\
n=34\end{array}$ & $\begin{array}{c}\text { Grupo controlo } \\
n=20\end{array}$ & $p$-value & $\begin{array}{l}\text { Magnitude } \\
\text { de efeito }\end{array}$ \\
\hline \multicolumn{5}{|l|}{ EGA, Med $\left[Q_{1} ; Q_{3}\right]$} \\
\hline Respeito pelo tecido & $4[3 ; 4]$ & $3[1,25 ; 4]$ & $0,018^{a, *}$ & 0,32 \\
\hline Tempo no movimento & $3[3 ; 4]$ & $3[1 ; 4]$ & $0,207^{a}$ & 0,17 \\
\hline Manuseamento dos instrumentos & $3[3 ; 4]$ & $3[1,25 ; 4]$ & $0,169^{\mathrm{a}}$ & 0,19 \\
\hline Treino de sutura & $4[3 ; 4]$ & $3[1 ; 4]$ & $0,188^{\mathrm{a}}$ & 0,18 \\
\hline Fluência do procedimento & $4[3 ; 4]$ & $4[1 ; 4]$ & $0,070^{\mathrm{a}}$ & 0,25 \\
\hline Conhecimento do procedimento & $4[3 ; 4]$ & $4[2,25 ; 4]$ & $0,135^{\mathrm{a}}$ & 0,20 \\
\hline Produto final & $4[3 ; 4]$ & $4[1 ; 4]$ & $0,483^{\mathrm{a}}$ & 0,10 \\
\hline Performance geral & $4[3 ; 4]$ & $4[2 ; 4]$ & $0,127^{\mathrm{a}}$ & 0,21 \\
\hline Pontuação total & $29,5[26 ; 32]$ & $28,5[10,5 ; 31,8]$ & $0,167^{a}$ & 0,19 \\
\hline \multicolumn{5}{|l|}{ Lista de verificação específica de procedimento, n (\%) } \\
\hline Técnica asséptica & $29(85,3 \%)$ & $14(70 \%)$ & $0,294^{b}$ & 0,18 \\
\hline Limpeza centrífuga da ferida duas vezes & $34(100 \%)$ & $16(80 \%)$ & $0,015^{\mathrm{b}, *}$ & 0,37 \\
\hline Escolha apropriada do tipo de fio de sutura & $21(61,8 \%)$ & $3(15 \%)$ & $0,001^{\mathrm{c}, *}$ & 0,45 \\
\hline Colocação de agulha no porta-agulhas & $29(85,3 \%)$ & $11(55 \%)$ & $0,014^{\mathrm{c}, *}$ & 0,33 \\
\hline Entrada e saída perpendicular do tecido & $33(97,1 \%)$ & $14(70 \%)$ & $0,008^{\mathrm{b}, *}$ & 0,39 \\
\hline Perfuração do tecido não inferior a $0,5 \mathrm{~cm}$ & $27(79,4 \%)$ & $12(60 \%)$ & $0,124^{c}$ & 0,21 \\
\hline Técnica de nó com porta-agulhas & $34(100 \%)$ & $13(65 \%)$ & $<0,001^{\mathrm{b}, *}$ & 0,50 \\
\hline Técnica de "nó de cirurgião" & $32(94,1 \%)$ & $13(65 \%)$ & $0,009^{b, *}$ & 0,38 \\
\hline Corte do fio de sutura com margens adequadas & $22(64,7 \%)$ & $6(30 \%)$ & $0,014^{\mathrm{c}, *}$ & 0,34 \\
\hline Técnica de remoção da sutura & $18(52,9 \%)$ & $2(10 \%)$ & $0,002^{\mathrm{c}, *}$ & 0,43 \\
\hline
\end{tabular}

a: teste de Mann-Whitney; b: teste exato de Fisher; c: teste do qui-quadrado.

*: significativo a $5 \%$

Pontuações EGA

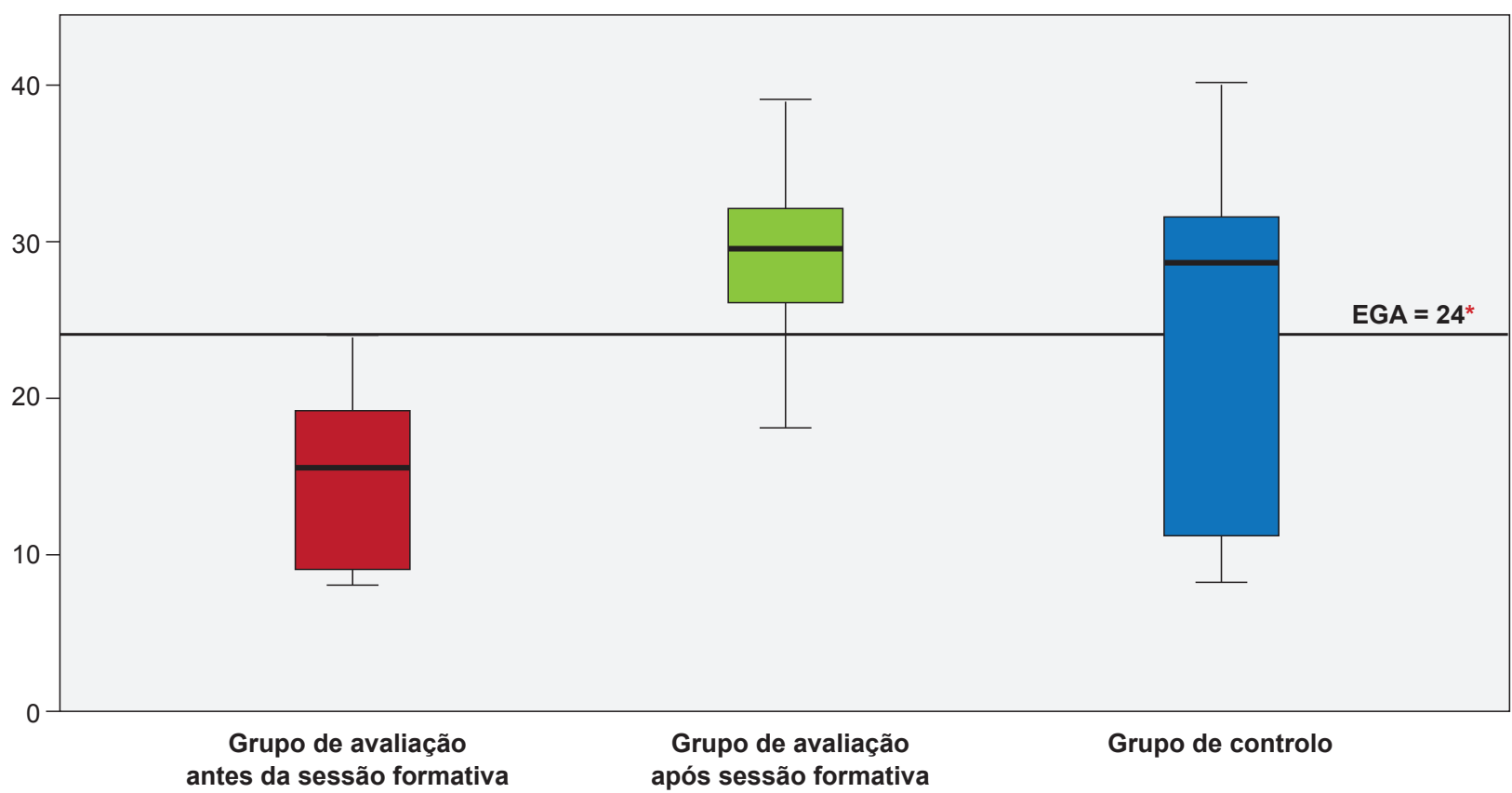

Figura 2 - Pontuações totais obtidas na escala global de avaliação (EGA) para o grupo de avaliação nos dois momentos (antes e após a sessão formativa) e para o grupo de controlo (que só teve um momento único de avaliação) *: Valor a partir do qual o avaliando é considerado competente na técnica. ${ }^{28}$ 
item RT apresentou diferenças estatisticamente significativas ( $p$-value $=0,018$ ) entre o GA ApF e o GC, sendo que o GA ApF obteve uma pontuação mediana mais elevada (4 vs 3), refletindo-se numa me média $(0,32)$. Para os restantes itens não foram encontradas quaisquer diferenças significativas nos dois grupos. O mesmo acontece para a PT da escala, isto é, apesar do GA ApF ter obtido uma PT mediana mais elevada $(29,5$ vs 28,5$)$, as distrubuições dos dois grupos não foram consideradas significativamente diferentes ( $p$-value $=0,167$ ), apresentando uma baixa me $(0,19)$. A Fig. 2 ilustra a PT da EGA para o GA nos dois momentos (AnF e ApF) e para o GC (um único momento de avaliação). É de salientar que o GA AnF se encontrava maioritariamente abaixo dos 24 pontos, valor a partir da qual se é considerado competente na técnica de sutura. É também de salientar que o GC apresenta uma dispersão superior ao GAApF, uma vez que a PT da EGA no GC varia de 8 a 40 pontos, enquanto que no GAApF varia de 18 a 39 pontos.

Quanto à LVEP, os únicos parâmetros para os quais não foram encontradas diferenças estatisticamente significativas foram a TA ( $p$-value $=0,294)$ e a PTNI ( $p$-value $=0,124)$, apresentando baixas me $(0,18$ e 0,21 , respetivamente). Para os restantes parâmetros, o GA obteve uma percentagem de realizações corretas bastante superiores ao GC, principalmente nos parâmetros EATFS (61,8\% vs $15 \%$; me $=0,45)$; TRS $(52,9 \%$ vs $10 \%$; me =0,43); TNPA
(100\% vs 65\%; me =0,50); CFSMA (64,7\% vs 30\%; me = $0,34)$ e CAPA $(85,3 \%$ vs $55 \%$; $m e=0,33)$. A Fig. 3 ilustra os resultados obtidos pela aplicação da LVEP nos dois grupos e, como é possível observar, em todos os procedimentos abrangidos pela lista, houve mais estudantes a realizá-los corretamente no GAApF do que no GC.

\section{DISCUSSÃO}

Tal como apresentado em outros estudos, 4,11,12 o programa de AAP implementado contribuiu para uma melhoria qualitativa e quantitativa significativa do conhecimento e $\mathrm{CT}$, abrangendo maior número de estudantes e com menores recursos da faculdade. A avaliação obtida pela EGA revelou uma evolução significativamente positiva nos estudantes AnF e ApF, assim como a LVEP também demonstrou um aumento do número de estudantes que realizaram corretamente os parâmetros da lista. Foi demonstrado noutro trabalho ${ }^{34}$ que uma única sessão de treino de CT, no currículo pré-graduado, pode, por si só, promover melhoria dessas competências e de motivação nos estudantes. Este aspeto foi também verificado neste estudo.

As técnicas em medicina são gestos diários de competências clínicas, suportadas por uma forte componente de conhecimentos científicos. Sendo o desenvolvimento de proficiência numa técnica um processo cumulativo e progressivo, ${ }^{33}$ o seu ensino deve iniciar-se ainda durante a formação pré-graduada. ${ }^{1,3}$ A metodologia de AAP para ensino

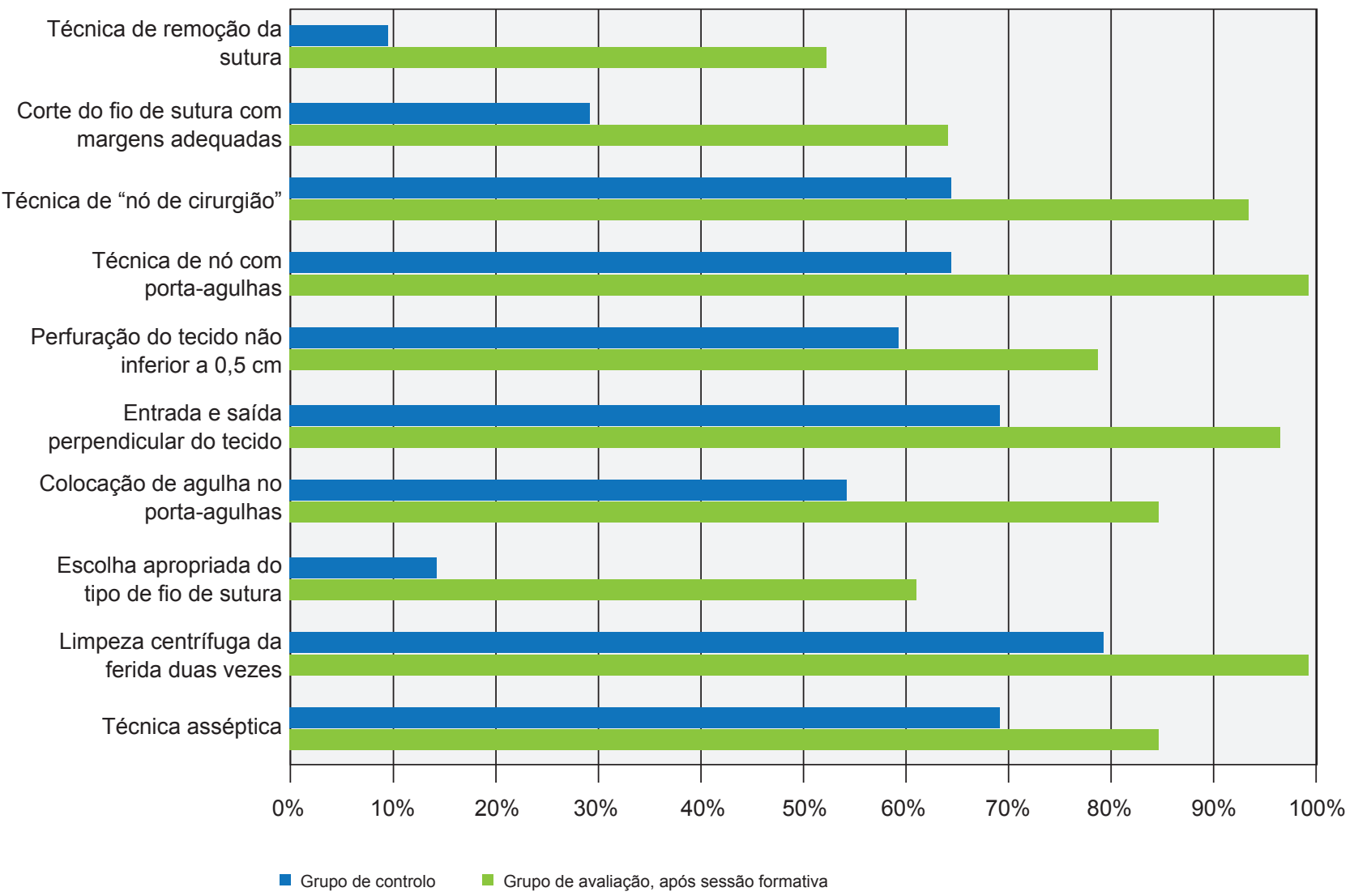

Figura 3 - Percentagens de estudantes do grupo de avaliação (após sessão formativa) e do grupo de controlo, que realizaram corretamente os procedimentos abrangidos pela lista de verificação 
de TCB permite rentabilizar recursos e chegar a um maior número de estudantes, preparando-os com competências pedagógicas e promovendo a sua competência e confiança. ${ }^{4,11,16,17}$

Poucos estudos avaliaram AAP em TCB $4,11,23,34,35$ e, ainda menos, através do instrumento OSATS. ${ }^{11}$ Os resultados obtidos neste trabalho foram idênticos aos existentes na literatura, ${ }^{11}$ embora os valores medianos da EGA, ApF, sejam superiores neste trabalho. Este resultado pode ser explicado pelo facto de, neste estudo, se tratar de estudantes já no final do terceiro ano, que iniciaram a avaliação AnF com valores de EGA mais altos, uma vez que a diferença entre pontuações AnF e ApF foi semelhante nos dois estudos. Relativamente à LVEP, ApF, obtiveram-se taxas de sucesso superiores a $70 \%$ para todos os parâmetros, à exceção dos seguintes: EATFS, CFSMA e TRS. Aliás, na LCF2x, todos os estudantes foram bem-sucedidos ApF. Estes resultados podem ser explicados pelo facto de serem técnicas simples, que encerram pouca complexidade cognitiva e, por isso, podem facilmente ser ensinadas por estudantes preparados para tal.

Quando comparada a PT na EGA do GA, ApF, com a do $G C$, verificou-se que não existem diferenças estatisticamente significativas entre os dois grupos. No entanto, existe uma maior dispersão de resultados nos estudantes do GC, o que pode significar que a formação proporcionada aos estudantes não é uniforme e consistente e, que depende, em grande medida, das oportunidades proporcionadas pelo contexto clínico, indo ao encontro de Davis et al. ${ }^{1}$ Este resultado é refererente a estudantes em final de formação (com todas as unidades curriculares concluídas), indo ao encontro do estudo de Grilo et $a l,{ }^{3}$ em que os recém-graduados identificam os procedimentos práticos, incluindo sutura, como uma das áreas em que se sentem menos competentes.

Quanto à percepção dos estudantes sobre o currículo e sobre a sessão, identificou-se que os estudantes avaliaram como negativo o grau de exposição académico a TCB, à semelhança de trabalhos prévios. ${ }^{1,3,4}$ Mais de 80 estudantes (> 90\%) concordaram que a sessão formativa contribuiu para desenvolver o seu conhecimento e CT em TCB. Esta perceção acompanha a evolução da performance ApF e está de acordo com um outro estudo semelhante, ${ }^{4}$ bem como a ideia de que a AAP em TCB pode, de facto, contribuir para uma melhoria de competências na técnica. ${ }^{4,11}$ Também mais de 80 estudantes (> 90\%) concordaram que a sessão formativa aumentou o nível de confiança e motivação no desempenho das técnicas, atribuindo pontuações superiores a um estudo idêntico anterior. ${ }^{4}$ Estes dados corroboram o que está descrito na literatura, nomeadamente nos estudos de Hill, ${ }^{12}$ Topping, ${ }^{13}$ Ten Cate ${ }^{16}$ e Bennet. ${ }^{35}$

Todos os parâmetros de qualidade da sessão foram classificados com uma pontuação mediana superior a 4 (escala de Likert 1 - 5), o que revela a satisfação dos estudantes com a qualidade da formação proporcionada, em concordância com o estudo de Preece et al. ${ }^{4}$ Quase todos os estudantes $(96 \%)$ sentiram que foram ensinados num ambiente relaxado que potenciou a sua aprendizagem. Este ambiente relaxado, que é referido por vários estudos como uma das caraterísticas e mais-valias da AAP, a que se associa uma maior congruência cognitiva e social, ${ }^{12,13,16,23,36}$ promove possivelmente níveis de aprendizagem mais elevados quando comparado com o ensino tradicional. ${ }^{34} \mathrm{De}$ facto, é conhecido que ambientes pouco relaxados aumentam os níveis de ansiedade, que podem ser prejudiciais à aquisição de conhecimentos e ao esclarecimento de dúvidas. ${ }^{36}$

A amostra utilizada no GA deste estudo foi selecionada aleatoriamente, com critérios de inclusão e exclusão bem definidos, o que diferencia este estudo de outros do género.4,11,12 No entanto, os resultados obtidos são concordantes com os de outros estudos.,12 $\mathrm{A}$ utilização do OSATS como instrumento de avaliação é um ponto deste trabalho a destacar, dado que este é considerado gold standard para avaliação objetiva de competências técnicas cirúrgicas. ${ }^{38}$ Optou-se que as avaliações ficassem a cargo de um só examinador para evitar algum grau de viés de examinador. A utilização de um método de avaliação estruturado, com parâmetros de avaliação bem definidos, pré-estabelecidos, e validados noutros contextos, pretendeu também mitigar alguns enviesamentos subjetivos.

Neste estudo, o tempo para avaliação de retenção foi reduzido (um mês), ficando a dúvida se os resultados se manteriam com um intervalo de tempo maior. Esta será uma questão de investigação para trabalho futuro. Como outras limitações referir que não foi efetuada análise de representatividade e apesar de ter sido obtido um tamanho de amostra satisfatório e com cálculo de magnitudes de efeito, poderia ter interesse estender o estudo às restantes escolas médicas. Para efeitos de maior validade dos dados, teria sido vantajosa a utilização de um questionário já validado. No entanto, os autores não encontraram nenhum na literatura existente.

Este estudo mostra que um programa de AAP, bem organizado, com estudantes dos últimos anos, pode ser uma mais-valia para o ensino de competências, sem prejuízo para os estudantes, à semelhança do que já foi concluído num estudo retrospetivo de grande escala. ${ }^{19} \mathrm{O}$ ambiente descontraído, a responsabilização dos mais velhos para uma das funções de se ser médico, a formação própria e dos seus pares (transmissão da experiência), o desenvolvimento de espírito de equipa e de solidariedade entre colegas, são valores desenvolvidos num programa deste tipo. ${ }^{16,20,36}$ Como refere Furmedge et $a l,{ }^{17}$ muitos destes estudantes podem já estar a desempenhar estas funções dentro dos seus núcleos de associativismo e, simplesmente beneficiariam de um suporte académico que asseguras$\mathrm{se}^{22}$ rigor ao conhecimento transmitido, alinhado com os objetivos curriculares da faculdade. É evidente que o envolvimento dos estudantes na construção do seu currículo e aprendizagem tem uma correlação significativa com os seus níveis de satisfação e, isso não pode nem deve ser menosprezado. ${ }^{17,16}$ Esta realidade fica demonstrada pelo 
facto de todos os estudantes referirem que voltariam a participar numa sessão de AAP, como formandos, e mais de $80(>90 \%)$ referirem mesmo que considerariam a possibilidade de participar como formadores. Facto que se encontra em consonância com um estudo parecido. ${ }^{35}$

A tendência revelada por estes resultados foi motivo para a criação na FMUC de uma unidade curricular opcional, denominada Ensino Assistido por Pares, que se iniciou no ano letivo de 2017/2018, à qual os estudantes têm aderido de forma entusiasta e cuja primeira avaliação será efetuada após três anos letivos. Esta unidade curricular é composta por três vertentes práticas (gestos clínicos básicos, TCB e suporte básico de vida) e uma pedagógica e destina-se a estudantes do quinto ano que irão ensinar os colegas mais novos.

\section{CONCLUSÃO}

Este programa de aprendizagem assistida por pares revelou-se promissor para a melhoria das competências técnicas cirúrgicas dos estudantes de medicina, com utilização de escassos recursos da faculdade e extensão a um número mais alargado de estudantes.

Os resultados encontrados neste estudo justificam a necessidade de aprofundar a investigação de modo longitudinal, bem como avaliar a transferência da aprendizagem para a prática clínica. Pode ser interessante estender o estudo a outras competências e domínios do saber.

\section{PROTECÇÃO DE PESSOAS E ANIMAIS}

Os autores declaram que os procedimentos seguidos estavam de acordo com os regulamentos estabelecidos pelos responsáveis da Comissão de Investigação Clínica e Ética e de acordo com a Declaração de Helsínquia da Associação Médica Mundial.

\section{CONFIDENCIALIDADE DOS DADOS}

Os autores declaram ter seguido os protocolos do seu centro de trabalho acerca da publicação de dados.

\section{CONFLITOS DE INTERESSE}

Os autores declaram não ter conflitos de interesse relacionados com o presente trabalho.

\section{FONTES DE FINANCIAMENTO}

Este projeto não recebeu qualquer apoio financeiro.

\section{REFERÊNCIAS}

1. Davis CR, Toll EC, Bates AS, Cole MD, Smith FC. Surgical and procedural skills training at medical school - a national review. Int J Surg. 2014;12:877-82.

2. Taylor I, Reed M, Kingsnorth A, Carlson G, Leaper D. Surgery in the undergraduate curriculum. Report by the Education and Professional Development Committee of the Society of Academic and Research Surgery (SARS). Royal Coll of Surg Engl. 2005;138-9.

3. Grilo Diogo P, Barbosa J, Ferreira MA. A Pilot Tuning Project-Based National Study on Recently Graduated Medical Students' SelfAssessment of Competences-The TEST Study. BMC Med Educ. 2015;15:226

4. Preece R, Dickinson EC, Sherif M, Ibrahim Y, Ninan AS, Aildasani L, et al. Peer-assisted teaching of basic surgical skills. Med Educ Online. 2015;20:275-9.

5. Victorino RM, Jollie C, McKimm J. O licenciado médico em Portugal. Lisboa: Faculdade de Medicina de Lisboa. 2005.

6. General Medical Council. Tomorrow's doctors - outcomes and standards for undergraduate medical education. London: GMC; 2009.

7. General Medical Council. Outcomes for graduates. London: GMC; 2015.

8. Costa P, Castelo HB, Alves CP, Pimentel J, Ribeiro V, Gomes MR, et al. Core graduate outcomes of Portuguese undergraduate medical education: guidelines for curriculum development surgery. Lisboa: Faculdade de Medicina de Lisboa; 2005.

9. Cumming A, Ross M. The Tuning Project for Medicine - learning outcomes for undergraduate medical education in Europe. Med Teach. 2007;29:636-41.

10. Grilo Diogo P, Moreira A, Coimbra A, Silva A, Martins A, Mendonça C, et al. Study on Portuguese medical schools' learning conditions: a national analysis on student satisfaction, student-tutor ratios and number of admissions. Acta Med Port. 2016;29:301-9.

11. Denadai R, Toledo AP, Oshiiwa M, Saad-Hossne R. Acquisition of suture skills during medical graduation by instructor-directed training: a randomized controlled study comparing senior medical students and faculty surgeons. Updates Surg. 2013;65:131-40.

12. Yu TC, Wilson NC, Singh PP, Lemanu DP, Hawken SJ, Hill AG. Medical students-as-teachers: a systematic review of peer-assisted teaching during medical school. Adv Med Educ Pract. 2011;2:157-72.

13. Topping KJ. The effectiveness of peer tutoring in further and higher education: a typology and review of the literature. High Educ. 1996;32:321-45.
14. Resnick PJ, MacDougall E. The use of senior medical students as preceptors in freshman clinical science. J Med Educ. 1976;51:763-5.

15. Ross MT, Cameron HS. Peer assisted learning: a planning and implementation framework: AMEE Guide No. 30. Med Teach. 2007;29:527-45.

16. Ten Cate O, Durning S. Peer teaching in medical education: twelve reasons to move from theory to practice. Med Teach. 2007;29:591-9.

17. Furmedge DS, Iwata K, Gill D. Peer-assisted learning - beyond teaching: how can medical students contribute to the undergraduate curriculum? Med Teach. 2014;36:812-7.

18. Nelson AJ, Nelson SV, Linn AM, Raw LE, Kildea HB, Tonkin AL. Tomorrow's educators...today? Implementing near-peer teaching for medical students. Med Teach. 2013;35:156-9.

19. Ten Cate O, Van De Vorst I, Van Den Broek S. Academic achievement of students tutored by near-peers. Int J Med Educ. 2012;3:6-13.

20. Ten Cate O. Perspective paper / Perspektive: peer teaching: from method to philosophy. Z Evid Fortbild Qual Gesundhwes. 2017;1278:85-7.

21. Saleh $M$, Sinha $Y$, Weinberg D. Using peer-assisted learning to teach basic surgical skills: medical students' experiences. Med Educ Online. 2013;18:210-65.

22. Yoong A, Mukundu Nagesh N, Rye DS, Devaraj V. Consultant led peer assisted learning model. Clin Teach. 2019;16:502-6.

23. Loda T, Erschens R, Loenneker H, Keifenheim KE, Nikendei C, Junne $\mathrm{F}$, et al. Cognitive and social congruence in peer-assisted learning - a scoping review. PLoS One. 2019;14:1-15.

24. Wadoodi A, Crosby JR. Twelve tips for peer-assisted learning: a classic concept revisited. Med Teach. 2002;24:241-4.

25. Royal College of Surgeons of England. Surgical skills for students Programme. RCS Education Copyright. 2015. [consultado 2016 jan 14]. Disponível em: https://www.rcseng.ac.uk/education-and-exams/ courses/search/surgical-skills-for-students.

26. Random.org [www.random.org]. Ireland: Randomness and Integrity Services Ltd. 2010. [consultado 2016 jan 14]. Disponível em https:// www.random.org.

27. Martin JA, Regehr G, Reznick R, Macrae H, Murnaghan J, Hutchison C, et al. Objective Structured Assessment of Technical Skill (OSATS) for surgical residents. Br J Surg. 1997;84:273-8.

28. Khan MS, Bann SD, Darzi AW, Butler PE. Assessing surgical skill using bench station models. Plast Reconstr Surg. 2007;120:793-800.

29. Denadai R, Saad-Hossne R, Todelo AP, Kirylko L, Souto LR. Low-fidelity 
bench models for basic surgical skills training during undergraduate medical education. Rev Col Bras Cir. 2014;41:137-45.

30. O'Connor HM, McGraw RC. Clinical skills training: developing objective assessment instruments. Med Educ. 1997;31:359-63.

31. Dubrowski A, MacRae H. Randomised, controlled study investigating the optimal instructor: student ratios for teaching suturing skills. Med Educ. 2006;40:59-63.

32. Sweller J. Working memory, long-term memory, and instructional design. J Appl Res Mem Cogn. 2015;5:360-7.

33. Dreyfus SE. The five-stage model of adult skill acquisition. Technol Society. 2004;24:177-81.

34. Lossing A, Groetzsch G. A prospective controlled trial of teaching basic surgical skills with 4th tear medical students. Med Teach. 1992;14:49-52.

35. Bennett SR, Morris SR, Mirza S. Medical students teaching medical students surgical skills: the benefits of peer-assisted learning. J Surg Educ. 2018;75:1471-4.

36. Ten Cate O, Durning S. Dimensions and psychology of peer teaching in medical education. Med Teach. 2007;29:546-52.

37. Friedlander MJ, Andrews L, Armstrong EG, Aschenbrenner C, Kass JS, Ogden $P$, et al. What can medical education learn from the neurobiology of learning? Acad Med. 2011;86:415-20.

38. Van Hove PD, Tuijthof GJ, Verdaasdonk EG, Stassen LP, Dankelman J. Objective assessment of technical surgical skills. $\mathrm{Br} \mathrm{J}$ Surg. 2010;97:972- 87 